\title{
El pez Trachelyopterus striatulus (Siluriformes: Auchenipteridae) como herramienta de muestreo de la entomofauna en un embalse tropical
}

\author{
Alejandra Filippo Gonzalez Neves dos Santos ${ }^{1}$, Francisco Racca-Filho ${ }^{2}$, Luciano Neves dos \\ Santos $^{3} \&$ Francisco Gerson Araújo ${ }^{2}$ \\ 1. Departamento de Zootecnia e Desenvolvimento Agro-Sócio-Ambiental Sustentável, Universidade Federal Fluminense, \\ Rua Vital Brasil, 64, Faculdade de Veterinária, Niterói, RJ - Brasil. CEP.: 24230-340; FAX: +55 21 33148205; \\ alejandrafilippo@hotmail.com \\ 2. Departamento de Biologia, Universidade Federal Rural do Rio de Janeiro, Antiga BR 465, Km 47, Seropédica, RJ - \\ Brasil. CEP: 23851-970; fraccafo@ufrrj.br; gerson@ufrrj.br \\ 3. Departamento de Biologia-NUPELIA, Universidade Estadual de Maringá, Av. Colombo, 5790, bloco H 90, Maringá, \\ PR - Brasil. CEP.: 87020-900; luciano.lep@gmail.com
}

Recibido 10-I-2009. Corregido 09-V-2009. Aceptado 09-VI-2009.

\begin{abstract}
The fish Trachelyopterus striatulus (Siluriforms: Auchenipteridae) used to sample insects in a tropical reservoir. The study of aquatic environments is sometimes difficult to do with normal sampling methods that use gears. Insectivorous fishes represent good users of these ecosystems and analyzing the aquatic organisms present in fish stomachs, is an alternative way to determine resource abundance and utilization. In this paper, the potential of Trachelyopterus striatulus as an insect sampler was examined through dietary analy-

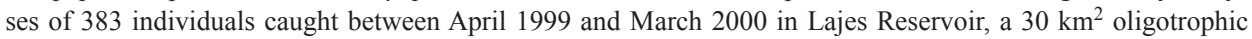
impoundment in Southeast Brazil. We estimated frequency of occurrence and Schoener's index of similarity. Diet changes among seasons and reservoir zones were addressed with DCA and ANOVA analyses. Its diet was $92.1 \%$ insects (ten orders and nine families). Hymenoptera (57.90\%), Odonata (39.76\%), Trichoptera (27.41\%), Ephemeroptera $(26.25 \%)$ and Coleoptera $(28.96 \%)$ were the most common groups. Highest insect occurrence and richness were recorded in autumn-summer, a period of greater rainfall and insect activity. Formicidae, the dominant prey item in all seasons, appeared to be especially important in spring, a season marked by shortness of food resources. Trichoptera and Ephemeroptera were the most consumed prey items in the other seasons. Highest insect occurrence and richness were recorded in the middle and upper reservoir zones, respectively. Trichoptera and Ephemeroptera prevailed in the upper zone, where small pristine rivers and tributaries are abundant, whereas Formicidae and Belostomatidae predominated in the lower and middle zones. Because of its abundance in many freshwater ecosystems of Brazil, the ubiquity of insects in its digestive tract and the low level of prey degradation, T. striatulus has potential as an insect sampler of Neotropical reservoirs. However, conventional sampling in Lajes Reservoir is necessary to compare the effectiveness of $T$. striatulus with other insect sampling methods. Rev. Biol. Trop. 57 (4): 1081-1091. Epub 2009 December 01.
\end{abstract}

Key words: Auchenipteridae, reservoir, trophic ecology, Brazil, insects.

Los insectos son el grupo de organismos terrestres más diversos del planeta, sin embargo sólo un diez por ciento de sus especies colonizan los ambientes acuáticos, destacándose los órdenes Ephemeroptera, Odonata, Plecoptera, Neuroptera (Megaloptera), Trichoptera, Lepidoptera, Diptera, Coleoptera y Hemiptera, siendo que los dos últimos poseen especies que desarrollan todo su ciclo de vida en el agua (McCafferty 1981).

La presencia, alóctona y autóctona, y la densidad de insectos en los ecosistemas acuáticos continentales, constituye una importante fuente de alimento en la dieta de peces de agua dulce. Sin embargo, uno de los importantes retos en la ecología trófica está en asociar 
los estudios sobre la dieta de peces con los recursos alimenticios disponibles. Cuando la obtención de muestras fidedignas de recursos tróficos acuáticos hechas en el ecosistema es complicada, los estudios sobre la dieta de los peces pueden ser más provechosos que los muestreos en el ecosistema (Winemiller 1989).

De esta manera, debido a las dificultades metodológicas para efectuar muestreos de insectos en orillas de ríos y lagos con vegetación cerrada, o en hábitats acuáticos complejos, como densos cinturones de macrófitos, los análisis del contenido estomacal de peces insectívoros aportarían informaciones importantes sobre la entomofauna. Los peces pueden acceder a determinados tipos de microhabitats acuáticos, los cuales muchas veces contienen organismos que son difíciles de capturar con los métodos de captura convencionales. Según Wooton (1990), los peces son, en general, buenos muestreadores del ambiente y los componentes de su dieta muestran lo que está disponible en el medio. No obstante, estudios con énfasis sobre el uso de peces como herramienta de muestreo de la entomofauna son todavía escasos.

El objetivo del presente estudio es realizar un levantamiento entomofaunístico en un embalse tropical, utilizando la especie Trachelyopterus striatulus (Siluriformes, Auchenipteridae), un pez insectívoro y de pequeña talla $(<30 \mathrm{~cm} \mathrm{LT})$ como herramienta de muestreo. Trachelyopterus striatulus es conocido como torito (español), singing catfish (inglés) o cumbaca (portugués) y está ampliamente distribuido en ríos y embalses de Sudamérica, extendiéndose desde Argentina hacia el Panamá (Nelson 1976). Aunque no sea una especie de interés comercial en las pesquerías, $T$. striatulus es una de las especies más abundantes en el embalse de Lajes (un lago artificial oligotrófico de $30 \mathrm{~km}^{2}$ ubicado en Río de Janeiro, Sureste de Brasil) donde representa aproximadamente $40 \%$ del total de las pesquerías experimentales ya realizadas (Duarte et al. 2002).

\section{MATERIAL Y MÉTODOS}

Área de estudio: El embalse de Lajes, Bra-

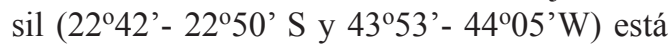

longitudinalmente distribuido en tres zonas, que obedecen a la clasificación general propuesta por Thornton et al. (1990) para ambientes represados: alta-es la cabecera, ubicada arriba del embalse, cerca de la desembocadura de los principales tributarios que lo abastecen, presenta las menores profundidades, con orillas menos inclinadas y a menudo colonizadas por macrófitos acuáticos; intermedia-ubicada entre las zonas alta y baja, es la región más ancha del embalse; y baja-situada cerca de la presa, con orillas inclinadas y de talud abrupto, mayores profundidades y poca disponibilidad de estructuras y refugios sumergidos.

Muestreo y análisis de los datos: La captura de los peces se llevó a cabo mensualmente en las tres zonas del embalse, desde abril de 1999 hasta marzo de 2000, utilizándose redes de enmalle simple $(30 \mathrm{mx} 3 \mathrm{~m})$, con 2.5 a $5.5 \mathrm{~cm}$ de tamaño de malla entre nudos opuestos y tiempo de permanencia de $12 \mathrm{~h}$. Los peces recogidos fueron acondicionados en recipientes isotérmicos con hielo, siendo después transferidos al laboratorio, donde fueron identificados, medidos, pesados y eviscerados para el posterior análisis del contenido estomacal de los individuos de T. striatulus.

Se utilizó una lupa estereoscópica para la determinación taxonómica del contenido estomacal siguiendo la clave de insectos propuesta por Pérez (1988), Merritt \& Cummim (1996), y a veces se contó con el apoyo de expertos, para llegar hasta el nivel taxonómico más bajo posible.

Se aplicó el método de Frecuencia de ocurrencia $(\% \mathrm{FO})$, el cual expresa la relación en porcentaje de estómagos con un determinado ítem alimenticio, y el número total de estómagos con alimento (Hyslop 1980). El nivel de dominancia de los insectos se estableció según la frecuencia de los taxones en el contenido estomacal: dominantes ( $\geq 15 \%)$; frecuentes (10$15 \%)$; y raros ( $\leq 10 \%)$ (Galina \& Hahn 2004).

También se analizó el porcentaje de similitud (\%PS) de Schoener (1968) según la ecuación: $\% \mathrm{PS}=100-0.5 \times \sum \mathrm{a}-\mathrm{b}$, donde: $\mathrm{a}=\% \mathrm{FO}$ de cada insecto en el muestreo $a ; y=\% F O$ 
de cada insecto en el muestreo b. Los valores superiores a $60 \%$ indican alta similitud en cuanto a la entomofauna. Los valores de $\% \mathrm{FO}$ y de \%PS fueron calculados de acuerdo con las variaciones temporales (estaciones del año) y espaciales (zonas baja, intermedia y alta).

Se utilizó un Análisis de Correspondencia Destendido-ACD, para identificar eventuales patrones temporales y espaciales de la entomofauna. Los resultados de los ejes retenidos y codificados en el ACD para cada estación del año y zonas del embalse, fueron comparados estadísticamente por un Análisis de Varianza (ANOVA). Cuando las medias fueron significativamente diferentes, se aplicó el test post-hoc HSD no balanceado al nivel de 95\% de confianza. Siempre que los supuestos estadísticos no fueron alcanzados, se utilizó el test de Kruskal-Wallis $(\alpha=0.05)$, y el test post-hoc para comparación múltipla de Kruskal-Wallis.

\section{RESULTADOS}

Se analizó el contenido estomacal de 383 individuos de T. striatulus, de los cuales 103 estómagos estaban vacíos. El ámbito de la longitud total fue de de 10.4-27.7 cm (media
15.4; error estándar $0.87 \mathrm{~cm}$ ) y el peso total varió entre 11.7 y $160.1 \mathrm{~g}$ (43.9; 0.97g). Doscientos ochenta individuos presentaron contenido estomacal, del cual un $92.1 \%$ fueron insectos y $7.9 \%$ representó otros organismos (peces, crustáceos, bivalvos, materia vegetal y arena). En el total, se registraron diez órdenes y nueve familias entre insectos acuáticos (A) y terrestres $(\mathrm{T})$. Los órdenes más frecuentes fueron $(\% \mathrm{FO})$ : Odonata $(37.1 \% \mathrm{~A} ; 2.7 \%$ $\mathrm{T}$ ), constituido principalmente por Aeshnidae (inmaduro); Trichoptera (27.4\% A), sobretodo Hydropsychidae; Ephemeroptera (26.3\% A); Hemiptera $(13.5 \% \mathrm{~A} ; 1.9 \% \mathrm{~T})$, representado principalmente por Belostomatidae; Hymenoptera $(57.9 \% \mathrm{~T})$, con dominio de Formicidae; y Coleoptera $(29.0 \%$ A), representado exclusivamente por Curculionidae. Los demás órdenes de insectos, correspondieron en conjunto a $6.2 \%$ A y $0.8 \% \mathrm{~T}(\mathrm{n}=258)$ (Fig. 1$)$.

Variaciones temporales: La presencia y dominancia de los insectos en el contenido estomacal de T. striatulus, separados por estaciones del año, se presentan en el Cuadro 1. La época de mayor diversidad y presencia de insectos consumidos fue el otoño, seguido del verano.

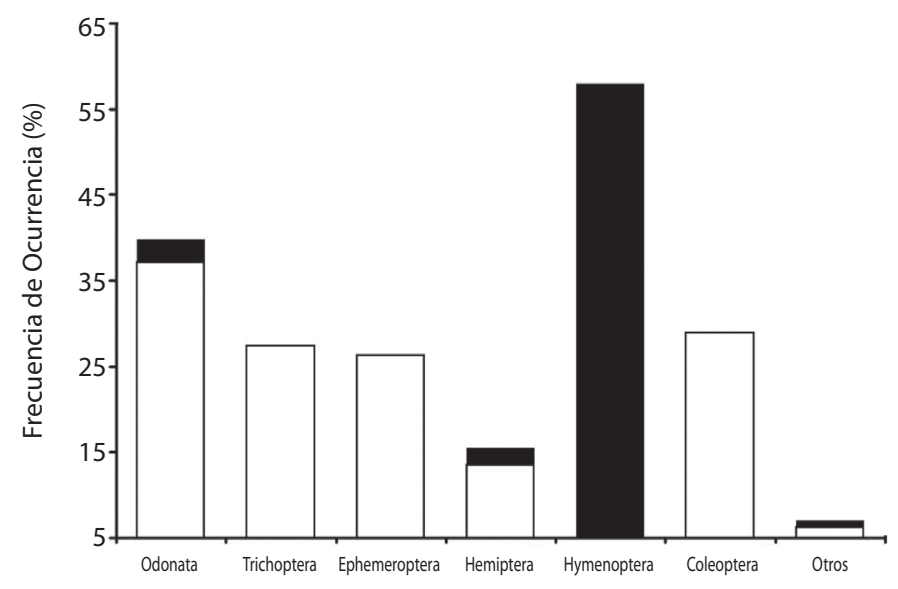

Fig. 1. Frecuencia de ocurrencia (\%) de los órdenes de insectos consumidos por Trachelyopterus striatulus en el embalse de Lajes (n=258). $\square=$ insectos acuáticos; $\square=$ insectos terrestres.

Fig. 1. Frequency of occurrence (\%) for the insect orders consumed by Trachelyopterus striatulus in Lajes reservoir ( $\mathrm{n}=258)$. $\square=$ aquatics insects; $\square=$ terrestrial insects. 
CUADRO 1

Frecuencia de ocurrencia $(\% F O)$ de los insectos presentes en la dieta de Trachelyopterus striatulus, según las estaciones del año en el embalse de Lajes, Brasil

TABLE 1

Frequency of occurrence (\%FO) by season for the insects recorded in the guts of Trachelyopterus striatulus in Lajes Reservoir, Brazil

\begin{tabular}{|c|c|c|c|c|}
\hline Grupos & $\begin{array}{l}\text { Otoño } \\
\mathrm{n}=106\end{array}$ & $\begin{array}{c}\text { Invierno } \\
\mathrm{n}=36\end{array}$ & $\begin{array}{c}\text { Primavera } \\
n=29\end{array}$ & $\begin{array}{c}\text { Verano } \\
\mathrm{n}=87\end{array}$ \\
\hline \multicolumn{5}{|l|}{ Hymenoptera } \\
\hline Formicidae & $* 50.47$ & $* 50.00$ & $* 82.76$ & $* 27.59$ \\
\hline Apidae & 12.15 & $* 19.44$ & 10.34 & 4.60 \\
\hline Vespidae & 2.80 & - & - & - \\
\hline \multicolumn{5}{|l|}{ Coleoptera } \\
\hline Curculionidae & $* 25.23$ & $* 25.00$ & $* 62.07$ & $* 24.14$ \\
\hline \multicolumn{5}{|l|}{ Odonata } \\
\hline Aeshnidae (inmaturo) & $* 41.12$ & $* 52.78$ & 10.34 & *34.48 \\
\hline Aeshnidae (adulto) & 4.67 & 2.78 & - & 1.15 \\
\hline \multicolumn{5}{|l|}{ Trichoptera } \\
\hline Hydropsychidae & $* 29.91$ & 11.11 & - & $* 40.23$ \\
\hline Ephemeroptera & *31.78 & $* 27.78$ & 6.90 & $* 25.29$ \\
\hline \multicolumn{5}{|l|}{ Hemiptera } \\
\hline Auchenorrhyncha & 1.87 & 2.78 & - & 2.30 \\
\hline Belostomatidae & $* 23.36$ & 8.33 & 13.79 & 3.45 \\
\hline Thysanoptera & - & - & 3.45 & - \\
\hline \multicolumn{5}{|l|}{ Blattodea } \\
\hline Blattidae & - & - & 3.45 & - \\
\hline \multicolumn{5}{|l|}{ Diptera } \\
\hline Chironomidae (larva) & 4.67 & - & - & 2.30 \\
\hline \multicolumn{5}{|l|}{ Orthoptera } \\
\hline Gryllidae & 4.67 & - & 3.45 & 3.45 \\
\hline Total acuático & 160.75 & 125.00 & 96.55 & 133.33 \\
\hline Total terrestre & 71.96 & 75.00 & 100.00 & 35.63 \\
\hline
\end{tabular}

Sin embargo, en primavera, los insectos acuáticos predominaron en la dieta de T. striatulus, obedeciendo la consiguiente secuencia: otoño (160.75\%FO), verano (133.33\%FO) e invierno $(125 \% \mathrm{FO})$. El índice de Schoener indicó una baja similitud en el consumo de insectos por T. striatulus entre la primavera y verano ( $\mathrm{PS}=45.90 \%$ ); primavera y otoño ( $\mathrm{PS}=55.29 \%$ ) y primavera e invierno ( $\mathrm{PS}=55.70 \%$ ) y alta similitud entre el verano y otoño (PS=80.96\%); verano e invierno (PS=75.90\%); y otoño e invierno ( $\mathrm{PS}=80.99 \%$ ).
Por presentar mayores autovalores y consecuentemente, explicar mejor la ordenación de las muestras codificadas por estaciones del año, los dos primeros ejes del ACD, fueron retenidos para confección y análisis del diagrama de ordenación (Fig. 2).

El eje 1 presentó autovalor de 0.6083 y Formicidae $(\mathrm{r}=0.487)$ fue la familia que más influenció la distribución de los muestreos del cuadrante positivo, mientras Hydropsychidae $(\mathrm{r}=-0.698)$ influenció la distribución de los muestreos del cuadrante negativo. El eje 2 


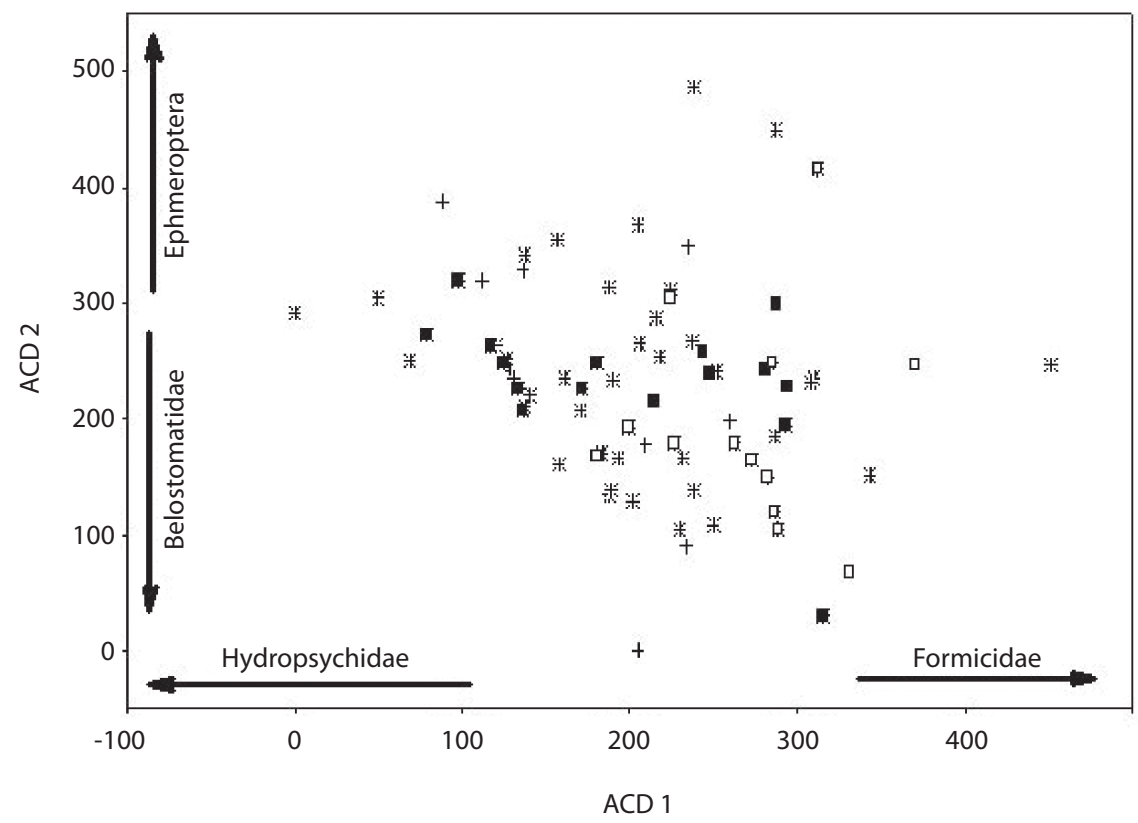

Fig. 2. Diagrama de ordenación-ACD, indicando la entomofauna en las estaciones del año. Las setas indican la contribución de los principales insectos descriptores de la ordenación. $*=$ otoño; $\mathbf{\square}=$ invierno; $\square=$ primavera; += verano

Fig. 2. DCA diagram indicating the seasonal variation of the insect families consumed by Trachelyopterus striatulus in Lajes reservoir, Brazil. Arrows indicate the contribution of the main descriptors insects of the ordination. $*=$ autumn; $\mathbf{\square}=$ winter; $\square=$ spring; += summer.

presentó un autovalor de 0.4479, y Ephemeroptera $(\mathrm{r}=0.419)$ fue el orden que más influenció en la distribución de los muestreos del cuadrante positivo, mientras la familia Belostomatidae $(\mathrm{r}=-0.499)$ influenció en la distribución de los muestreos del cuadrante negativo.

Los valores del eje 1 del ACD no alcanzaron los supuestos de normalidad (ShapiroWilk's $=0.950, \mathrm{p}<0.001$ ) y de homocedasticidad (Test de Levene $=16.75, p<0.001$ ), para realizar ANOVA, siendo detectados problemas evidentes en la dispersión de los valores alrededor de la media, que interfieren significativamente en los resultados del ANOVA. Así, fue aplicado el análisis de Kruskal-Wallis. El test detectó diferencias estacionales significativas (KruskalWallis $=34.169, \mathrm{p}<0.001$ ), y el test post-hoc para comparación múltiple identificó valores más elevados en la primavera, con relación a las demás estaciones del año $(\mathrm{p}<0.05)$ (Fig. 3).
Posteriormente fueron analizados los supuestos para la aplicación del ANOVA sobre las medias de los resultados del eje 2 del ACD. Los resultados del eje 2 del ACD tampoco alcanzaron los supuestos de normalidad (Shapiro-Wilk's=0.973, p<0.001), y de homocedasticidad (Test de Levene $=3.59, \mathrm{p}=0.014$ ), por lo que se aplicó el test de Kruskal-Wallis. El test identificó la existencia de diferencias estacionales significativas en los resultados del eje 2 (Kruskal-Wallis $=20.278, \mathrm{p}<0.001$ ), y el test de post-hoc para comparación múltiple revela que la primavera presentó valores más bajos con relación a las demás estaciones del año $(\mathrm{p}<0.05)$ (Fig. 3).

De acuerdo con la distribución de los muestreos a lo largo de los dos primeros ejes del ACD, hay evidencias al nivel de 95\% de confianza, de que la entomofauna se diferenció entre las estaciones del año, con mayores 

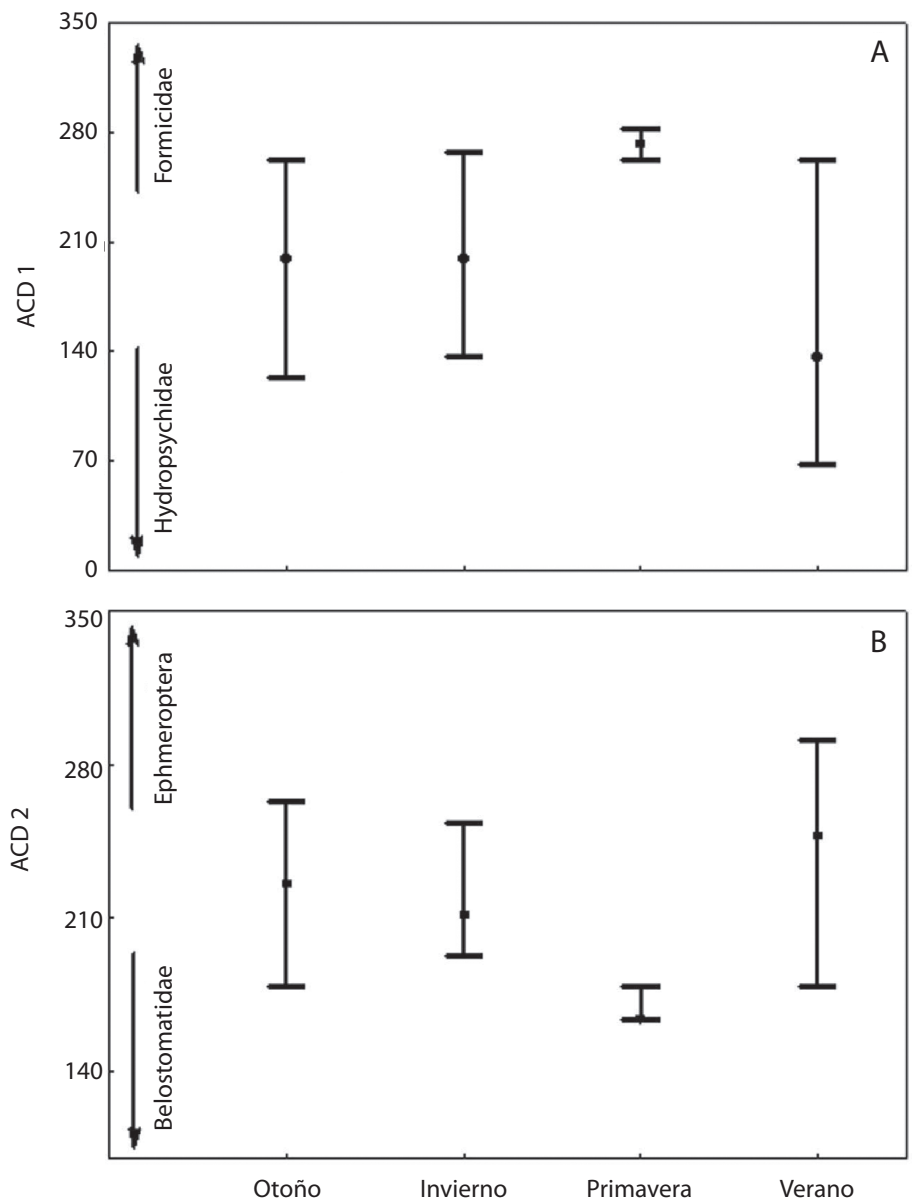

Fig. 3. A) Mediana y cuartiles $25-75 \%$ de los resultados del eje 1 del ACD para las estaciones del año. Las setas indican la contribución de los principales insectos descriptores del eje 1 de la ordenación. B) Mediana y cuartiles 25-75\% para los resultados del eje 2 del ACD para las estaciones del año. Las setas indican la contribución de los principales insectos descriptores del eje 2 de la ordenación.

Fig. 3. A) Median and 25-75\% quartiles of the DCA's axis 1 scores by season. Arrows indicate the contribution of the main descriptor insects for the DCA axis 1 scores. B) Median and 25-75\% quartiles of the DCA's axis 2 scores by season. Arrows indicate the contribution of the main descriptor insects for the DCA axis 2 scores.

presencias de Formicidae y de Belostomatidae en la primavera, mientras Hydropsychidae y Ephemeroptera predominaron en las demás estaciones del año.

Variaciones espaciales: La presencia y dominancia de los insectos por zonas del embalse se presenta en el Cuadro 2. La zona intermedia y alta del embalse, presentan mayor diversidad y abundancia de insectos en la dieta de T. striatulus, respectivamente. Los insectos acuáticos predominaron en todas las zonas, exhibiendo mayores frecuencias de ocurrencia en la zona alta (170.23\%FO), que la baja $(111.32 \% \mathrm{FO})$ y que la intermedia $(106.76 \% \mathrm{FO})$. Se observó una baja similitud 
CUADRO 2

Frecuencia de ocurrencia (\% FO) de los insectos presentes en la dieta de Trachelyopterus striatulus en las zonas del embalse de Lajes, Brasil

TABLE 2

Frequency of occurrence $(\% F O)$ by reservoir zones for the insects recorded in the guts of Trachelyopterus striatulus in Lajes reservoir, Brazil

\begin{tabular}{|c|c|c|c|}
\hline Grupos & $\begin{array}{l}\text { Baja } \\
n=53\end{array}$ & $\begin{array}{l}\text { Intermedia } \\
\mathrm{n}=74\end{array}$ & $\begin{array}{c}\text { Alta } \\
\mathrm{n}=131\end{array}$ \\
\hline \multicolumn{4}{|l|}{ Hymenoptera } \\
\hline Formicidae & *56.60 & $* 70.27$ & *29.00 \\
\hline Apidae & 9.43 & *17.57 & 6.87 \\
\hline Vespidae & 1.89 & 1.35 & 0.76 \\
\hline \multicolumn{4}{|l|}{ Coleoptera } \\
\hline Curculionidae & $* 20.75$ & $* 47.30$ & *22.14 \\
\hline \multicolumn{4}{|l|}{ Odonata } \\
\hline Aeshnidae (inmaturo) & $* 37.74$ & *34.32 & $* 44.27$ \\
\hline Aeshnidae (adulto) & 3.77 & 1.35 & 3.05 \\
\hline \multicolumn{4}{|l|}{ Trichoptera } \\
\hline Hydropsychidae & 7.55 & 5.41 & $* 48.09$ \\
\hline Ephemeroptera & $* 22.64$ & 8.11 & *38.17 \\
\hline \multicolumn{4}{|l|}{ Hemiptera } \\
\hline Auchenorrhyncha & 1.89 & 4.05 & 0.76 \\
\hline Belostomatidae & $* 16.98$ & 13.51 & 12.21 \\
\hline Thysanoptera & - & 1.35 & - \\
\hline \multicolumn{4}{|l|}{ Blattodea } \\
\hline Blattidae & - & 1.35 & - \\
\hline \multicolumn{4}{|l|}{ Diptera } \\
\hline Chironomidae & 1.89 & 2.70 & 3.05 \\
\hline \multicolumn{4}{|l|}{ Orthoptera } \\
\hline Gryllidae & 3.77 & 5.41 & 2.29 \\
\hline Total acuático & 111.32 & 106.76 & 170.23 \\
\hline Total terrestre & 73.58 & 97.30 & 40.45 \\
\hline
\end{tabular}

de la entomofauna entre la zona alta e intermedia ( $\mathrm{PS}=55.67 \%$ ), mientras, una alta similitud fue observada entre las zonas baja y alta (PS $=77.51 \%$ ) y entre las zonas baja e intermedia ( $\mathrm{PS}=74.35 \%)$.

Para analizar la entomofauna por zonas del embalse, se utilizó un procedimiento análogo al de las estaciones del año y, al utilizar los mismos datos, los resultados de los ejes y valores fueron los mismos, diferenciándose solamente las muestras codificadas (Fig. 4).

Una vez que los valores del eje 1 del ACD no alcanzaron los supuestos del ANOVA de normalidad (Shapiro-Wilk's $=0.950, \mathrm{p}<0.001$ ) y de homocedasticidad (Test de Levene $=8.171$, $\mathrm{p}<0.001$ ), se aplicó el Kruskal-Wallis. El test detectó diferencias significativas para la entomofauna entre las zonas del embalse (KruskalWallis $=66.101, \mathrm{p}<0.001$ ), y el test de post-hoc para comparación múltiple reveló valores más elevados para las zonas baja e intermedia $(\mathrm{p}<0.05)$ (Fig. 5).

Posteriormente fueran analizados los supuestos para la aplicación del ANOVA para las medias de los valores del eje 2 de la ACD. Los resultados del eje 2 no alcanzaron los supuestos de normalidad (ShapiroWilk's $=0.973, p<0.001$ ), pero alcanzaron los de homocedasticidad (Test de Levene $=1.541$, $\mathrm{p}=0.216$ ). Así, se aplicó el ANOVA teniéndose en cuenta, que el supuesto de normalidad no fue considerado un problema muy grave que interfiriera significativamente en los resultados de la estadística paramétrica. Fueron detectadas diferencias espaciales significativas para la entomofauna (ANOVA, $\mathrm{F}=8.755, \mathrm{p}<0.001$ ), mientras el test post-hoc HSD no balanceado, confirmó al nivel de $95 \%$ de confianza $(\mathrm{p}<0.05)$, de que la zona alta presentó mayores valores que las zonas baja e intermedia en el eje 2 (Fig. 5).

De esta manera, se concluye que la dieta de T. striatulus se diferenció entre las zonas del embalse, con presencias elevadas de Hydropsychidae y Ephemeroptera en la zona alta, mientras Formicidae y Belostomatidae predominaron en las zonas baja e intermedia.

\section{DISCUSIÓN}

Aunque el espectro del muestreo fuera pequeño, fue posible obtener una gran diversidad de insectos en el embalse de Lajes e inferir sobre la importancia de este grupo como recurso alimenticio para T. striatulus, así como, probablemente, para otras especies de peces insectívoros presentes en el ambiente, tales como 


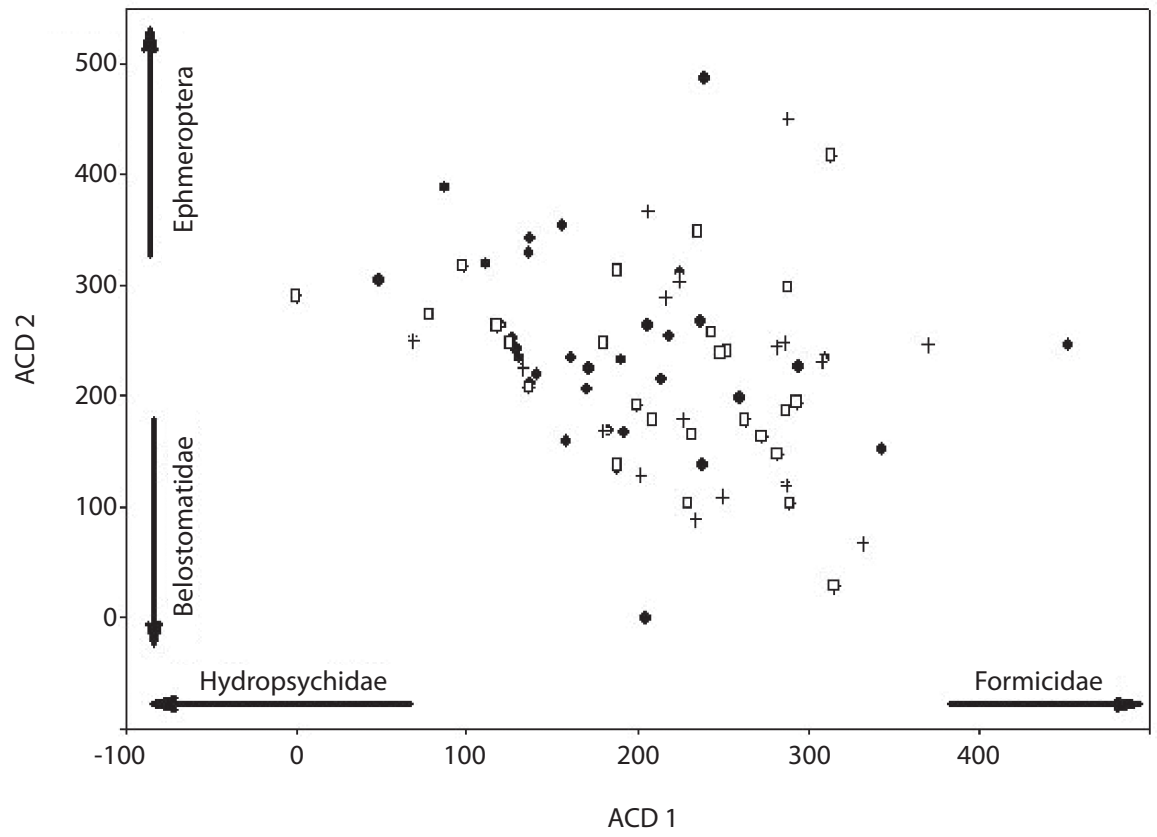

Fig. 4. Diagrama de ordenación-ACD, indicando la entomofauna en las zonas del embalse. Las setas indican la contribución de los principales insectos descriptores de la ordenación. $\square=$ zona baja; += zona intermedia; $>$ zona alta.

Fig. 4. DCA diagram, indicating the spatial variation of the insect families consumed by Trachelyopterus striatulus in Lajes reservoir, Brazil. Arrows indicate the contribution of the main descriptors insects of the ordination. $\square=$ lower zone; += middle zone; $=$ upper zone.

Astyanax cf. bimaculatus, Astyanax parahybae, Oligossarcus hepsetus, Metynnis maculatus, entre otros (Araújo \& Santos 2001).

Los invertebrados acuáticos, principalmente los insectos, en diferentes fases de desarrollo, son elementos frecuentes en la dieta de varias especies de peces de agua dulce (Bronmark 1994). En el presente trabajo, la mayor diversidad y presencia de insectos fueron observadas en el otoño y en el verano, estaciones que corresponden, en parte, al periodo de lluvias y de mayor actividad de la entomofauna (reproducción, alimentación entre otras). Según Russo et al. (2002) la ausencia de temporadas secas prolongadas y lluvias bien distribuidas a lo largo del año, pueden garantizar abundancia de alimento durante todo el año para la ictiofauna, una vez que garantizan la entrada de materia orgánica proveniente de la vegetación marginal, que es fuente de alimento para gran parte de los invertebrados acuáticos.

Entre los grupos de insectos muestreados, Hymenoptera, representado principalmente por Formicidae, fue considerado el ítem dominante en todas las estaciones del año y zonas del embalse, siendo consumido, en especial, en la primavera y en la zona baja e intermedia. Ese resultado puede estar relacionado a una preferencia alimenticia de $T$. striatulus por estos organismos, o puede ser un indicativo de versatilidad alimentaria presentada por esta especie. Tupinambás et al. (2007), señalan que algunas especies de peces tienen preferencias alimentarias por determinados invertebrados, independiente de su abundancia y presencia en el ambiente. 

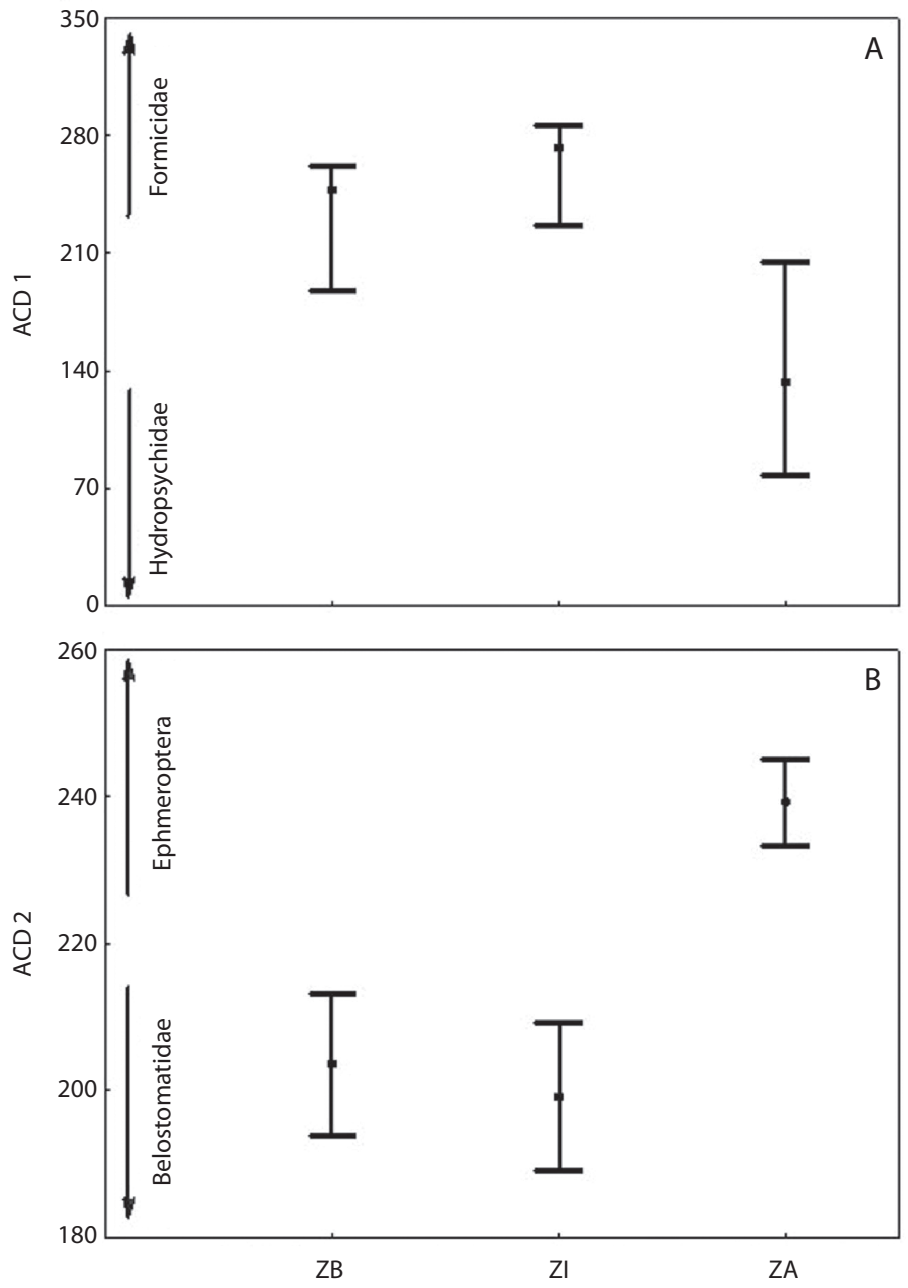

Fig. 5. A) Mediana y cuartiles $25-75 \%$ de los resultados del eje 1 del ACD para las zonas del embalse. Las setas indican la contribución de los principales insectos descriptores del eje 1 de la ordenación. B) Media \pm error estándar para los resultados del eje 2 del ACD para las zonas del embalse. Las setas indican la contribución de los principales insectos descriptores del eje 2 de la ordenación. $\mathrm{ZA}=$ zona alta; $\mathrm{ZI}=$ zona intermedia; $\mathrm{ZB}=$ zona baja.

Fig. 5. A) Median and 25-75\% quartiles of the DCA's axis 1 scores extracted to reservoir zones. Arrows indicate the contribution of the main descriptor insects for the DCA axis 1 scores. B) Means \pm standard error of the DCA's axis 2 scores extracted to reservoir zones. Arrows indicate the contribution of the main descriptor insects for the DCA axis 2 scores. ZA= upper zone; $\mathrm{ZI}=$ intermediate zone; $\mathrm{ZB}=$ lower zone.

En la primavera, T. striatulus presentó una baja similitud en la dieta con relación a las demás estaciones. En este período, $T$. striatulus presentó una menor variedad y frecuencia de insectos en los estómagos, siendo especialista en determinados tipos alimenticios, como Formicidae y Belostomatidae, los cuales podrían estar reflejando la disponibilidad alimentaria en aquel periodo. Según Tupinambás et al. (2007), las actividades antrópicas pueden afectar la comunidad de insectos en el ecosistema acuático, lo que puede justificar en parte, 
la plasticidad trófica de muchos peces de agua dulce ante de las variaciones estocásticas del ambiente.

Con respecto a las zonas del embalse, se registró mayor diversidad y Frecuencia de presencia de insectos en las zonas intermedia y alta respectivamente. Según Ward (1992) la composición de los insectos acuáticos en regiones lóticas esta influenciada por la mayor velocidad del flujo, presencia de vegetación ribereña y textura del sedimento, mientras en las regiones lénticas, la hipoxia y/o anoxia del hipolimnio y las mayores profundidades determinan las menores diversidades de organismos bentónicos, siendo la densidad de algunos grupos muy variada en este tipo de ambiente, lo que probablemente justifica los resultados registrados.

Lowe-McConnell (1987) resalta la importancia de invertebrados terrestres como recurso alimenticio para muchos peces y pondera que existe una clara sucesión linear de fuentes de alimentos en ambientes lóticos. Desde esta perspectiva, era de esperarse que los T. striatulus que habitan la zona alta, región que comprende las cabeceras del embalse de Lajes, dependieran primariamente de alimentos de origen alóctono, como Formicidae. En contraposición, la mayor frecuencia de Formicidae fue registrada en la zona intermedia, ambiente más amplio, profundo, desestructurado y menos turbio, donde el ciclo de nutrientes es menor debido la sedimentación, y consecuentemente, los recursos más disponibles de manera general son de origen autóctono (Thornton et al. 1990).

Trichoptera y Ephemeroptera fueron dominantes en la zona alta y, con excepción de la primavera, presentaron frecuencias elevadas a lo largo de todas las estaciones del año. Russo et al. (2002) y McCafferty (1981), señalan que estos grupos de insectos viven enterrados en fondos arenosos o rocosos, exigiendo ambientes bien oxigenados para su supervivencia. Según Russo et al. (2002) y Norris \& Georges (1993) la calidad biológica de los ecosistemas acuáticos continentales puede ser evaluada por las comunidades bióticas que los habitan. De esta manera, se especula que las importantes participaciones de Trichoptera y Ephemeroptera en la dieta de $T$. striatulus en la zona alta, no solo puede ser un indicativo de la buena calidad del agua del embalse de Lajes, corroborando con las informaciones de Santos et al. (2004), así como, puede ser reflejo de las características peculiares de la propia zona (bióticas y abióticas) que favorecen el desarrollo de estas especies.

Por fin, se concluye que T. striatulus presenta un potencial interesante como herramienta para muestrear la entomofauna del embalse de Lajes, contribuyendo para el conocimiento de la bioecología de los insectos y la ecología trófica de los organismos acuáticos presentes en este ambiente. Sin embargo, informaciones complementarias sobre la disponibilidad de la entomofauna, son esenciales para cuantificar la importancia de estos recursos en la cadena alimentaria de este ecosistema.

\section{AGRADECIMIENTOS}

El presente trabajo fue posible gracias al apoyo de CAPES-Coordenação de Aperfeiçoamento de Pessoal de Nível Superior, y al financiamiento obtenido mediante el proyecto PISCES convenio entre LIGHT S.A. y UFRRJ-Universidade Federal Rural do Rio de Janeiro, Brasil. A Luis Alberto Espínola y José Antonio Arenas Ibarra por sus observaciones y revisiones en el español del manuscrito.

\section{RESUMEN}

El objetivo de este trabajo fue hacer un levantamiento
entomofaunístico, en un embalse Tropical, utilizando como
organismo indicador al pez Trachelyopterus striatulus.
Fue analizada la dieta de 383 T. striatulus, muestreados
desde abril de 1999 hasta marzo de 2000 . La Frecuencia de
presencia y el porcentaje de similitud de Schoener fueron
aplicados para analizar la dieta. Para identificar variaciones
de la entomofauna entre las estaciones del año y las zonas
del embalse fueron aplicadas estadísticas univariadas y
multivariadas. La dieta estuvo compuesta principalmente
por insectos ( $92.14 \%$ ) siendo los más frecuentes (\%FO):
Hymenoptera (57.90\%), Odonata (39.76\%), Trichoptera
(27.41\%), Ephemeroptera (26.25\%) y Coleoptera (28.96\%).
Formicidae fue dominante en todas las estaciones del año,


en especial en la primavera, mientras, Trichoptera y Ephemeroptera fueron más consumidos en las demás estaciones del año. En la zona alta, predominaron Trichoptera y Ephemeroptera, mientras en las zonas baja e intermedia predominaron Formicidae y Belostomatidae. T. striatulus presenta potencial como muestreador de la entomofauna contribuyendo para el conocimiento de la biología y ecología de los insectos y la ecología trófica de los organismos acuáticos.

Palabras clave: Auchenipteridae, embalse, ecología trófica, Trachelyopterus striatulus, Brasil, insectos

\section{REFERENCIAS}

Araújo, F.G. \& L.N. Santos. 2001. Distribution and composition of fish assemblages in Lajes reservoir, Rio de Janeiro, Brazil. Braz. J. Biol. 61: 563-576.

Bronmark, C. 1994. Effects of trench and perch on interations in a freshwater, benthic food chain. Ecology 775: 1818-1828.

Duarte, S., C.B. Caetano, R.N., Vicentini \& F.G. Araújo. 2002. Distribuição e abundância relativa de cumbaca Trachelyopterus striatulus Steindachner no reservatório de Lajes. Rio de Janeiro, Brasil. Rev. Bras. Zoo. 19: 925-933.

Galina, A.B. \& N.S. Hahn. 2004. Atividade de forrageamento de Triportheus spp. (Characidae, Triportheinae) utilizada como ferramenta de amostragem da entomofauna, na área do reservatório de Manso, MT. Rev. Bras. Zoociências 6: 81-92.

Hyslop, E. J.1980. Stomach contents analysis-A review of methods and their application. J. Fish Biol. 17: 411-429.

Lowe-Mcconnell, R.H. 1987. Ecological studies in tropical fish communities. University Press, Cambridge, Inglaterra.

McCafferty, W.P. 1981. Aquatic entomology: the fishermen's and ecologist's illustred guide to insects and their relatives. Jones \& Bartlett, Boston, EEUU.

Merritt, R.W. \& K.W. Cummim. 1996. Ecology and distribution of aquatic insects, p. 74-86. In R.W. Merrit \&
K.W. Cummim (eds.). An introduction to the aquatic insects of North America. Kendall \& Hunt Publishing, Nueva York, EEUU.

Nelson, J.S. 1976. Fishes of the world. John Wiley, Nueva York, EEUU.

Norris, R.H. \& A. Georges. 1993. Analysis and interpretation of benthic macroinvertebrates surveys, p. 234-286. In D.M. Rosenberg \& V.H. Resh (eds.). Freshwater biomonitoring and benthic macroinvertebrates. Chapman Hall, Nueva York, EEUU.

Péréz, G.R. 1988. Guía para el estudio de los macroinvertebrados acuáticos del departamento de Antioquia. FEN Colombia y Conciencias, Bogotá, Colombia.

Russo, M.R., A. Ferreira \& R.M. Dias. 2002. Disponibilidade de invertebrados aquáticos para peixes bentófagos de dois riachos da bacia do rio Iguaçu, Estado do Paraná, Brasil. Act. Scient. 24: 411-417.

Santos, A.F.G.N., L.N. Santos \& F.G. Araújo. 2004. Water level influences on body condition of Geophagus brasiliensis in a Brazilian Reservoir. Neotrop. Ichthyol. 2: 151-156.

Schoener, T.H. 1968. The Anolis lizards of bimini: resource partitioning in a complex fauna. Ecology 49: 704726.

Thornton, K.W., B.L. Kimmel \& F.E. Paynne. 1990. Reservoir limnology: ecological perspectives. Interscience Publication, Nueva York, EEUU.

Tupinambás, T. H., M. Callisto \& G.B. Santos. 2007. Benthic Macroinvertebrate assemblage structure in two headwather streams, South Eastern, Brazil. Rev. Bras. Zoo. 24: 887 - 897 .

Ward, J.V. 1992. Aquatic insect ecology: 1. Biology and habitat. John Wiley \& Sons, Inc. Nueva York, EEUU.

Winemiller, K.O. 1989. Ontogenetic diet shifts and resource partitioning among piscivorous fishes in the Venezuelan llanos. Environ. Biol. Fish 26: 177-199.

Wooton, R.L. 1990. Ecology of teleost fishes. Chapman \& Hall, Londres, Inglaterra. 
